\title{
EPILEPSY AND FEBRILE CONVULSIONS
}

The risk of epilepsy after febrile convulsions was determined in a national population based study in the United Kingdom and reported from the Department of Paediatrics, Addenbrooke's Hospital, Cambridge and the Institute of Child Health, University of Bristol, England. Of 16,004 neonatal survivors born during 1 week in April 1970 and enrolled in the British Birth Survey, 398 (2.7\% of those followed) had at least 1 febrile convulsion by 10 years of age. The ratio of simple to complex febrile seizures was 4 to 1 . Those with complex febrile convulsions had a significantly greater incidence of febrile seizure recurrences than the simple febrile seizure group. Prophylactic anticonvulsants were prescribed for longer than a month in only $14 \%$ of patients; the seizure was simple in 26 and complex in 14. Afebrile seizures developed in 13 patients $(3.4 \%)$ and $9(2.3 \%)$ had epilepsy or recurrent afebrile seizures. Complex febrile convulsions and especially focal febrile convulsions were predictive of epilepsy. Afebrile complex partial seizures occurred in $9.4 \%$ of children with prolonged febrile convulsions. Of 14,278 children in this study who had no febrile convulsions, $53(0.4 \%)$ developed epilepsy by the age of 10 years (Verity CM, Golding J. Risk of epilepsy after febrile convulsions: a national cohort study. BMJ Nov 30, 1991; 303:1373-6). (Correspondence: Dr. Verity, Department of Paediatrics, Addenbrooke's Hospital, Cambridge CB2 2QQ, England.)

COMMENT. Complex febrile convulsions (longer than 15 minutes, focal, or multiple) accounted for $20 \%$ of the febrile seizures in this study, an incidence identical with that reported by pediatricians in the United States (Millichap JG. Clinical EEG. 1991; 22:5-12). In contrast, complex and simple febrile seizures are seen with equal frequency in pediatric neurology practice in the U.S. (Millichap JG, Colliver JA, Pediatr Neurol. 1991; $\underline{7}: 243-248)$. Compared to the United Kingdom where prophylactic anticonvulsants are used infrequently ( $14 \%$ of patients), in the United States long-term phenobarbital is prescribed by $89 \%$ of pediatric neurologists for the prevention of complex febrile seizures and by $43 \%$ for simple febrile seizures. Parental anxiety was a factor in the prescription of anticonvulsants by $67 \%$ of respondents to a questionnaire. Despite the publicity regarding adverse effects on behavior and cognition, phenobarbital therapy is still favored by the majority of pediatric neurologists in the U.S., particularly in the prevention of complex febrile seizures. The recent Seattle study (Farwell JR et al. N Engl J Med 1990; 322:364-9) failed to convince members of the Child Neurology Society that phenobarbital has any long-term persistent adverse effects on cognition and they are skeptical of the claim that phenobarbital is ineffective in the control of febrile seizures. The viewpoint stated in an editorial (Robinson RJ, BMJ Nov 30, 1991; 303: 1345-6) that prophylactic anticonvulsants are rarely needed and have no long term benefit in febrile convulsions is shared by only $17 \%$ of pediatric neurologists in the United States. 
Several factors might explain the transatlantic differences in the management of febrile seizures: 1) a greater availability and reliance on rectal diazepam for use by parents in the U.K., 2) geographic considerations and the greater distances to hospitals for emergency room treatment of the acute seizure in rural areas of the U.S., 3) the apparent importance of parental anxiety and physician concern for litigation in the U.S. Therapeutic guidelines may be helpful but the decision to treat must be made on an individual basis. When further and more carefully evaluated, perhaps the EEG will prove of value and receive attention in febrile seizure management.

\section{THE EEG AND FEBRILE SEIZURES}

The relationship between clinical characteristics and EEG classification in 676 children with febrile seizures was examined at the University Pediatric Clinic, Skopje, Yugoslavia. The initial EEG was classified as paroxysmally abnormal (spike wave complexes, generalized or focal, or spikes) in $22 \%$. Focal seizures and those that lasted more than 15 minutes were more often associated with abnormal EEGs. Multiple seizures in the first 24 hours was not related to risk of specific paroxysmal abnormalities. The leading predictors of initial paroxysmal EEG abnormality were 1) older age at the time of EEG and 2) the number of previous febrile seizures. Motor abnormality before the index seizure and focal seizure had less predictive value. The rate of EEG abnormality in children with no previous seizures was $18 \%$ whereas in those with 4 or more previous seizures it was $63 \%$ (Sofijanov $\mathrm{N}$ et al. Febrile seizures: clinical characteristics and initial EEG. Epilepsia Jan/Feb 1992; 33:52-57). (Reprints: Dr. K. B. Nelson at NIH, Federal Building, Room 700, Bethesda, MD 20892, U.S.A.)

COMMENT. Age at the time of the EEG, the number of seizures experienced and a focal first febrile seizure were all predictive of a paroxysmal EEG abnormality. In children more than 4 years of age abnormal EEGs were found in $51 \%$. Further follow-up of this population based sample should allow correlation of the initial EEG findings and the occurrence of subsequent afebrile seizures and epilepsy. In a questionnaire survey of pediatricians and pediatric neurologists in the U.S.A., the EEG was used in determining the need for long-term phenobarbital prophylactic therapy for febrile seizures by $51 \%$ and $45 \%$, respectively. Paroxysmal EEG abnormalities were more frequent in children 5 years of age and older than in younger age groups, and the incidence of EEG abnormalities in records of patients who developed afebrile seizures was 5 times that observed in children with febrile seizures alone. Paroxysmal tracings were more frequent in patients with prolonged or focal febrile seizures than in patients with short simple seizures (Millichap JG, Clinical EEG. 1991; 22:5-12. Idem, Febrile convulsions, 1968, McMillan, New York). 